\title{
Validation of the Korean version of the walking impairment questionnaire in patients with peripheral arterial disease
}

\author{
Chanjoong Choi ${ }^{1,2, *}$, Taeseung Lee ${ }^{1,3, *}$, Seung-Kee Min ${ }^{1,2}$, Ahram Han ${ }^{1,2}$, Song-Yi Kim ${ }^{1,2}$, Sang-il Min ${ }^{1,2}$, \\ Jongwon $\mathrm{Ha}^{1,2}$, In Mok Jung ${ }^{1,4}$ \\ ${ }^{1}$ Department of Surgery, Seoul National University College of Medicine, Seoul, ${ }^{2}$ Department of Surgery, Seoul National University \\ Hospital, Seoul, ${ }^{3}$ Department of Surgery, Seoul National University Bundang Hospital, Seongnam, ${ }^{4}$ Department of Surgery, Seoul \\ Metropolitan Government - Seoul National University Boramae Medical Center, Seoul, Korea
}

\begin{abstract}
Purpose: Intermittent claudication is the most common early symptom of peripheral arterial occlusive disease. Walking impairment questionnaire (WIQ) is a short, inexpensive, easy-to-complete questionnaire to assess intermittent claudication and can provide data of usual walking. The purpose of this study is to validate the new Korean version of WIQ.

Methods: Total 51 patients with claudication were enrolled. While 4 patients were dropped out, 47 patients with claudication into were divided groups based on the treatment received: surgery $(n=33)$ and medication $(n=14)$. The surgery group was subdivided into the bypass $(n=13)$ and intervention $(n=20)$ groups. WIQ score, ankle-brachial index $(A B I)$, and treadmill test scores were assessed initially and after 12 weeks.

Results: The WIQ scores were significantly correlated with ABI and pain-free walking distance (PFWD) and maximum walking distance (MWD) in all groups (except for MWD in the intervention group). Speed and stair-climb scores (2 WIQ domains) were well correlated with ABI, PFWD, and MWD. Distance scores were mostly correlated with ABI, PFWD, and MWD in all groups except $A B I$ in the bypass and intervention groups and MWD in the bypass group. Reproducibility was observed in all groups (intraclass correlation coefficient $>0.8$ ).

Conclusion: The Korean version of the WIQ is valid and reproducible, and can be effectively used to assess Korean patients with intermittent claudication.
\end{abstract}

[Ann Surg Treat Res 2017;93(2):103-109]

Key Words: Korea, Walking, Surveys and questionnaires, Peripheral arterial disease, Intermittent claudication

\section{INTRODUCTION}

Intermittent claudication (IC) is a walking-related impairment involving specific ischemic pain of the lower extremity, as a result of peripheral arterial occlusive disease (PAD). This PAD symptom specifically worsens after exercise such as walking or stair-climbing, although it usually disappears after a short rest period $[1,2]$. Assessments of the walking ability of PAD patients are important both during daily life and in the hospital. Hence, there is a need for a measurement method that can be easily used by patients to determine whether the disease severity has worsened [3-9].

The conventional test for IC usually involves the treadmill exercise test, wherein the distance of walking is measured until the initiation of pain or pain intolerance develops. This treadmill test is considered to be one of the most important
Received November 15, 2016, Revised February 1, 2017,

Accepted February 24, 2017

\section{Corresponding Author: In Mok Jung}

Department of Surgery, Seoul Metropolitan Government - Seoul National University Boramae Medical Center, 20 Boramae-ro 5-gil, Dongjak-gu, Seoul 07061, Korea

Tel: +82-2-870-2272, Fax: +82-2-870-3863

E-mail: sboy5240@gmail.com
*These authors contributed equally to this study and should be considered co-first authors

Copyright (c) 2017, the Korean Surgical Society

(c) Annals of Surgical Treatment and Research is an Open Access Journal. All articles are distributed under the terms of the Creative Commons Attribution NonCommercial License (http://creativecommons.org/licenses/by-nc/4.0/) which permits unrestricted non-commercial use, distribution, and reproduction in any medium, provided the original work is properly cited. 
objective tests [10]. However, it does not represent the walking ability of patients during daily life, and is impossible to perform without treadmill equipment or medical professionals. Moreover, this test is not practical in epidemiologic research. Therefore, Regensteiner, Hiatt, and others developed the walking impairment questionnaire (WIQ), which makes it simple and easy for patients to self-assess their walking ability $[11,12]$.

The WIQ measures walking distance, speed, and the ability to climb stairs. The WIQ can objectively measure PAD symptoms, and its use has been recommended in the Trans-Atlantic InterSociety Consensus [13]. The WIQ has been translated into several languages, including Dutch, German, Italian, Spanish, Swedish, Japanese, and Chinese, and is used worldwide [1420]. Translation of this questionnaire into the native language of the patients is essential for accurate assessment. Hence, a Korean version of the WIQ is important to evaluate Korean PAD patients. In the present study, we aimed to develop the Korean version of the WIQ and to validate its use among Korean PAD patients.

\section{METHODS}

\section{Patient enrollment}

We consecutively enrolled Korean patients diagnosed with PAD at 3 hospitals in 1 medical college. The inclusion criteria were as follows: age of 19-80 years; chronic symptoms of IC; ankle-brachial index (ABI) of <0.9; if ABI was abnormally high (>1.3), then toe-brachial index of $<0.7$; ability to perform treadmill test involving $12 \%$ gradient and $2.4-\mathrm{km} / \mathrm{hr}$ speed; informed consent for participating in the study. The exclusion criteria were as follows: critical limb ischemia with pain at rest or ischemic ulcers; ankle blood pressure $<40 \mathrm{mmHg}$; claudication induced by other causes such as spinal stenosis, arthritis, or cerebrovascular disease; high risk of acute coronary syndrome during the treadmill test; and anticipated noncompliance. The patients were treated either by medication, endovascular intervention, or open bypass based on the decision of the vascular surgeons. All participants eligible and willing to attend gave their written informed consent prior to their enrollment.

This study was approved by the Institutional Review Board of the Seoul National University Hospital (approval number: 1207-025-416).

\section{Korean version of the WIQ}

The WIQ was translated in compliance with Beaton's guidelines while respecting "the process of cross-cultural adaptation of self-report measures" [21]. First, the original English questionnaire was translated to the Korean version by three Korean bilingual translators. Thereafter, the translated
Korean version was translated back to the English version by another three bilingual translators whose native language was English. After discussion among the authors, the final Korean version was selected. Examples of scoring and samples of main questions that were used in this version of the WIQ are presented in Supplementary material 1.

\section{Study design for validation of WIQ}

We aimed to validate the Korean version of WIQ, by determining the reproducibility of the WIQ scores and its correlation with other objective indices such as ABI or treadmill test scores. The enrolled patients were divided into groups according to the treatment received: surgery and medication group. The surgery group was further divided into the bypass and intervention groups, for subgroup analysis. The objective indices of the patients included ABI, initial claudication distance, and maximum walking distance (MWD). All components of WIQ, including distance, speed, and stairclimbing scores were also analysed.

The ABI and treadmill tests, along with WIQ questionnaire scoring, were performed at baseline (Fig. 1). These tests and the questionnaire were again administered after 3 months. At initial stage of the study, it is planned that the data of the surgery group, including the bypass and intervention groups, would be analysed as one group like the medication group by using the paired t-test. Thereafter, the surgery group was split into the bypass and the intervention groups due to different severity of surgical site pain in early stage between the open surgery and the minimal invasive intervention. To test for reproducibility, patients were again administered the WIQ questionnaire at 1 month after treatment. A period of 1 month was considered to be sufficient time for the patients to forget the initial questionnaire data, but was not believed to be sufficient to exhibit the results of medication. However, in the surgery group, major clinical changes may be evident during

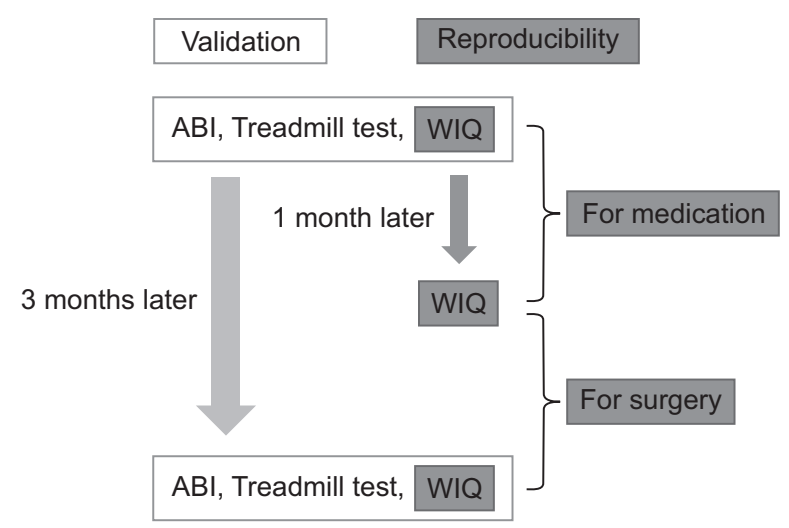

Fig. 1. Study flowchart for testing for validity and reproducibility. $\mathrm{ABI}$, ankle-brachial index; WIQ, walking impairment questionnaire. 
the first month itself, and hence, 1- and 3-month data were used to determine reproducibility. The intraclass correlation test was used to assess reproducibility. An intraclass correlation coefficient (ICC) between 0.8 and 1 , between 0.6 and 0.79 , and $<0.6$ was considered to very reliable, moderately reliable, and not reliable, respectively. Sample size was set as size in previous study with other language [22] and using G*Power software (version 3.0.10, Faul et al., Germany) that calculated required sample size was 34 in 2 tailed t-test with effect size 0.5 , alpha error 0.05 , and power (1-beta error) 0.8. Statistical computations were performed using SAS 9.3 (SAS Institute Inc., Cary, NC, USA), for the 1-way analysis of variance, paired t, repeatedmeasures analysis of variance, and Pearson correlation tests, as well as the IBM SPSS Statistics ver. 20.0 (IBM Co., Armonk, NY, USA) for the intraclass correlation test.

\section{RESULTS}

\section{Study population}

From March 2013 to May 2014, we enrolled 51 Korean PAD patients (Fig. 2). Four patients were excluded from the study after enrollment; the reasons included treatment for angina pectoris in 1 patient and loss to follow-up in 3 patients; 2 of
Table 1. Patient demographics

\begin{tabular}{|c|c|c|c|}
\hline Variable & $\begin{array}{l}\text { Bypass } \\
(\mathrm{n}=13)\end{array}$ & $\begin{array}{l}\text { Intervention } \\
\quad(\mathrm{n}=20)\end{array}$ & $\begin{array}{l}\text { Medication } \\
\quad(\mathrm{n}=14)\end{array}$ \\
\hline Age (yr) & $63.7 \pm 11.1$ & $66.9 \pm 9.9$ & $66.4 \pm 9.9$ \\
\hline Male sex & $12(92.3)$ & $19(95)$ & $14(100)$ \\
\hline \multicolumn{4}{|l|}{ Risk factors } \\
\hline Diabetes & 2 & 4 & 3 \\
\hline Hypertension & 5 & 14 & 9 \\
\hline Smoking & 6 & 8 & 7 \\
\hline Hyperlipidaemia & 6 & 1 & 0 \\
\hline Cardiovascular & 3 & 3 & 2 \\
\hline Cerebrovascular & 1 & 1 & 0 \\
\hline \multicolumn{4}{|l|}{ Site of disease } \\
\hline \multicolumn{4}{|l|}{ Aorto-iliac } \\
\hline Occlusion & 4 & 3 & 0 \\
\hline Severe stenosis & 4 & 8 & 5 \\
\hline \multicolumn{4}{|l|}{ Femoro-popliteal } \\
\hline Occlusion & 3 & 5 & 4 \\
\hline Severe stenosis & 7 & 5 & 7 \\
\hline \multicolumn{4}{|l|}{ Infrapopliteal } \\
\hline Occlusion & 1 & 2 & 0 \\
\hline Severe stenosis & 0 & 0 & 0 \\
\hline
\end{tabular}

Values are presented as mean \pm standard deviation, number $(\%)$, or number.

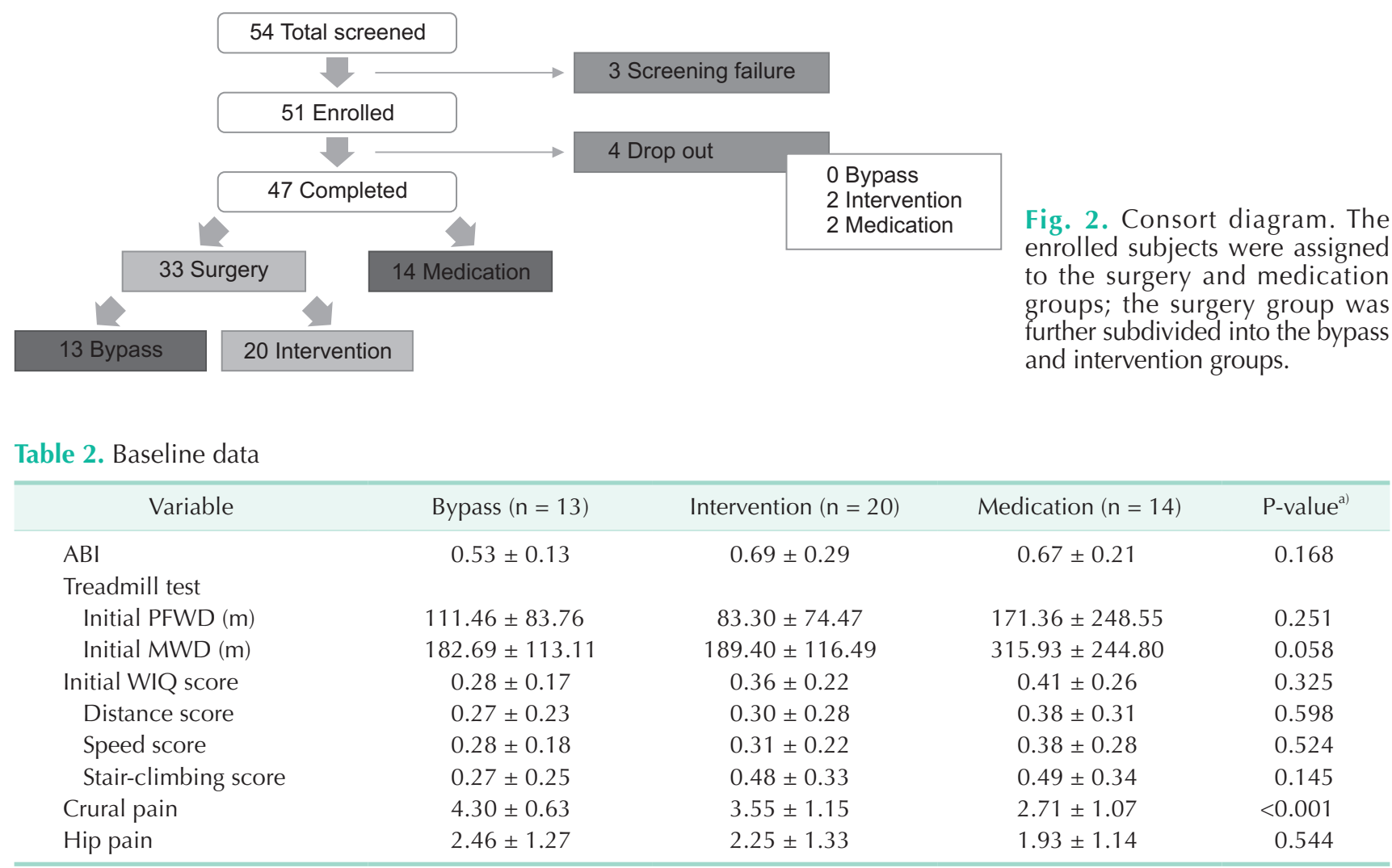

Values are presented as mean \pm standard deviation.

$\mathrm{ABI}$, ankle-brachial index; PFWD, pain-free walking distance; MWD, maximum walking distance; WIQ, walking impairment questionnaire.

${ }^{a)}$ One-way analysis of variance test. 
these patients were from the intervention group, and the other 2 were from the medication group. Finally, a total of 47 patients (13 from the bypass group, 20 from the intervention group, and 14 from the medication group) completed the follow-up. The patient demographics are summarised in Table 1. The mean age of the patients was $65.9 \pm 10.1$ years, and did not significantly differ among the 3 treatment groups $(P=0.665)$. Male sex was dominant in all the groups. The affected sites were determined during initial radiologic imaging. In the surgery group, the disease was more often occlusive and involved an aorto-iliac lesion, as compared to the medication group, although this did not significantly differ $(\mathrm{P}=0.210)$.
The ABI and treadmill test scores were obtained as objective data, whereas the initial WIQ scores were obtained as subjective data at baseline after informed consent was obtained (Table 2). There were no differences throughout the baseline data among the treatment groups. The degree of crural and hip pain was recorded initially and was also assessed along with the conventional WIQ score in order to reduce bias based on disease location and to represent the subjective symptoms that directly influence claudication but do not correspond with the radiologic results. Hip pain did not differ among the treatment groups, and all the patients experienced greater pain at the crural site than at the hip during the claudication period. Only
$A B I$

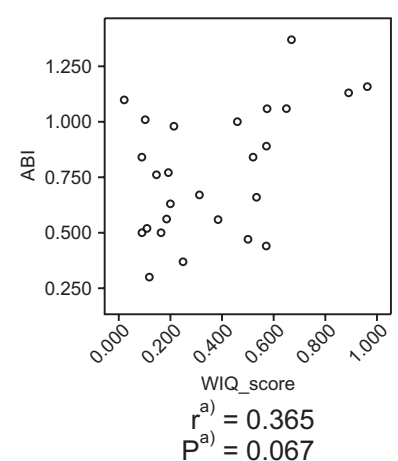

Bypass

$(n=13)$

$P^{\mathrm{a})}=0.067$

Treadmill test
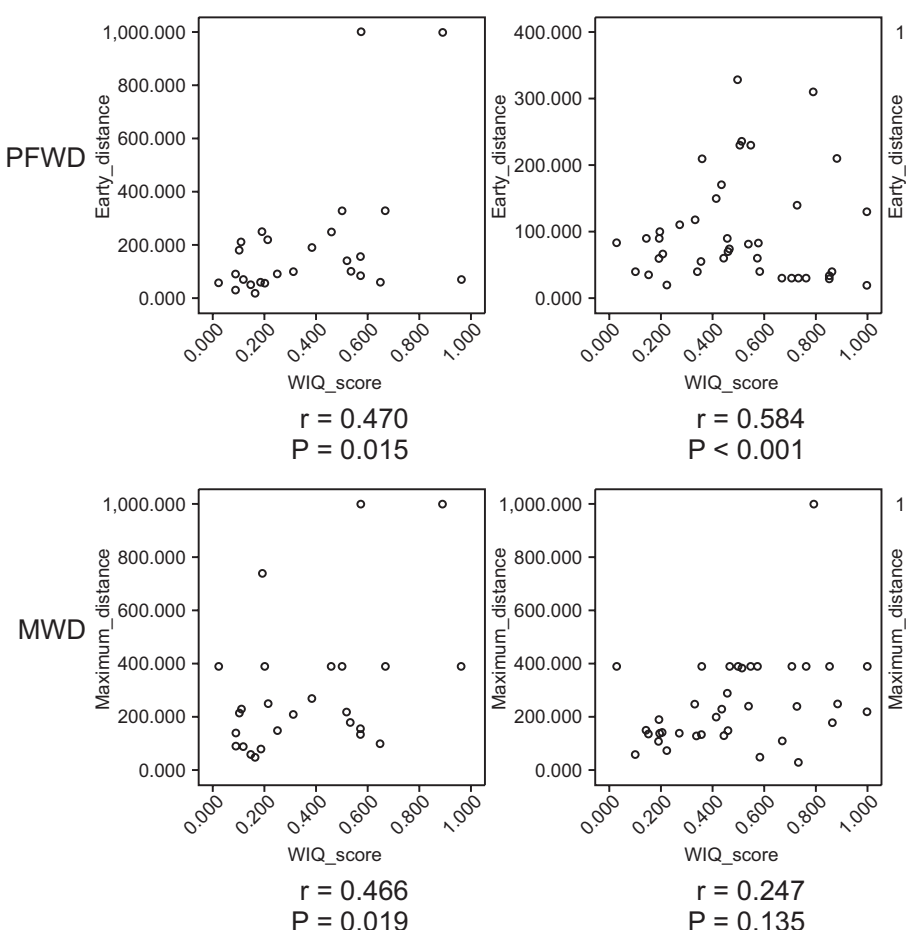

Intervention

$(n=20)$

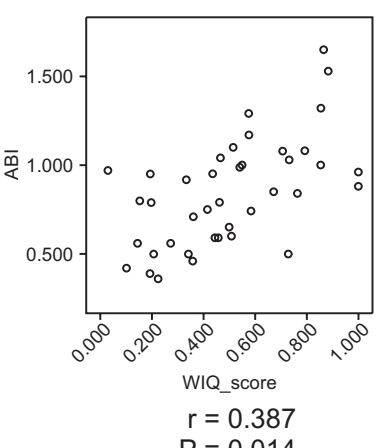

$\begin{aligned} r & =0.387 \\ P & =0.014\end{aligned}$
Surgery $^{\text {b) }}$

$(n=33)$

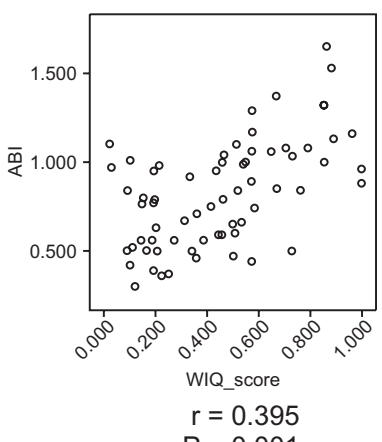

$P=0.001$

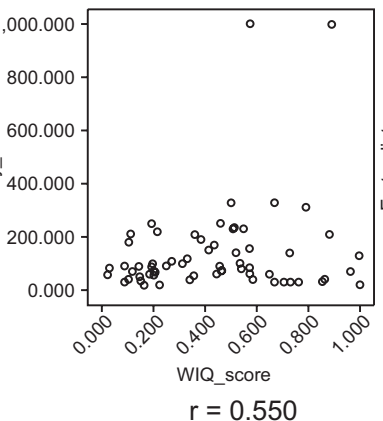

$P<0.001$

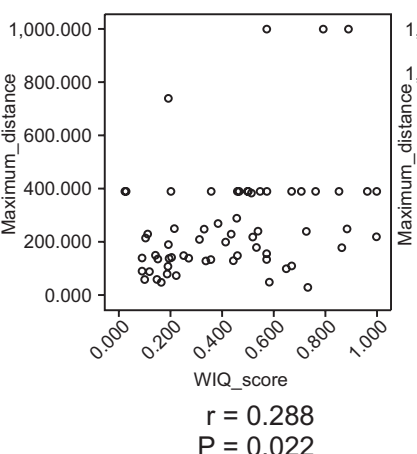

Medication

$(n=14)$

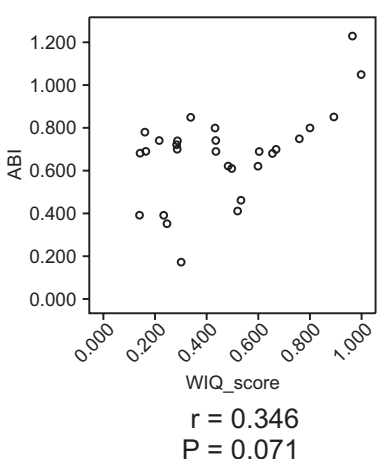

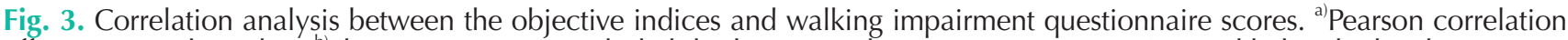

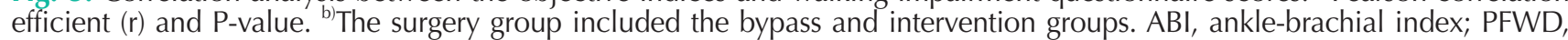
pain-free walking distance; MWD, maximum walking distance. 
the degree of crural pain exhibited a significant difference among groups $(\mathrm{P}<0.001)$.

\section{Assessment of validity using correlation}

Correlations between the objective indices (ABI, initial PFWD, and MWD) and the WIQ score are presented using a scatter plot (Fig. 3). The WIQ score was significantly correlated with the objective indices in all the treatment groups, except with MWD in the intervention group. However, the MWD score in the surgery group was well correlated with the objective indices.

The WIQ score included fragmentary scores, which were determined via detailed questions in the questionnaire. These fragmentary scores can also reflect the objective indices in each treatment group. The speed and stair-climbing scores showed a greater correlation with the objective indices (Table 3). Moreover, the distance score was correlated with all the objective indices, except with ABI in bypass and intervention groups and with MWD in the bypass group. The distance score was significantly correlated with ABI in the surgery group.

\section{Reproducibility}

The ICCs between the 1- and 3-month WIQ scores of the bypass and intervention groups were $0.82(\mathrm{P}=0.003)$ and $0.90(\mathrm{P}<0.001)$, respectively. The ICC between the baseline and 1-month scores of the medication group was $0.84(\mathrm{P}=$ 0.001). Based on the abovementioned definitions of reliability, it appeared that this version of the WIQ was reproducible, regardless of the treatment type, and that it is very reliable.

\section{DISCUSSION}

In the present study, we aimed to compare the usefulness of the WIQ in different clinical settings, including patients who received medication alone or those who underwent surgery including open bypass or interventional treatment. As the patients who received bypass surgery tended to complain about greater difficulty in walking during the early postoperative period, compared to patients who received interventional treatment, we subdivided the surgery group patients into the bypass and intervention groups. However, the bypass and intervention groups did not show any significant difference in the change in WIQ score according to time $(\mathrm{P}=0.380$ in repeated-measures analysis of variance test); moreover, the baseline data was not significantly different between these groups (Table 2).

All treatments led to clinical improvements in WIQ scores, $\mathrm{ABI}, \mathrm{PFWD}$, and MWD during the follow-up period. Between baseline and 3 months after treatment, the WIQ score improved by $0.43 \pm 0.20$ in the bypass group ( $\mathrm{P}<0.001), 0.42$ \pm 0.26 in the intervention group $(\mathrm{P}<0.001)$, and $0.24 \pm 0.22$ in the medication group $(P=0.002)$, each by paired t-test. The improvement in the WIQ score was significantly higher in the surgery group than in the medication group $(\mathrm{P}=0.045)$. The objective indices-including ABI, PFWD, and MWD-were

Table 3. Correlation analysis between the objective indices and each domain score of the WIQ

\begin{tabular}{|c|c|c|c|c|c|}
\hline Score & $*$ & Bypass & Intervention & Surgery ${ }^{\text {a) }}$ & Medication \\
\hline \multirow[t]{2}{*}{$\mathrm{ABI}$ - distance score } & $r$ & 0.296 & 0.280 & 0.243 & 0.368 \\
\hline & $P$ & 0.142 & 0.080 & 0.049 & 0.054 \\
\hline \multirow[t]{2}{*}{ Speed score } & $r$ & 0.656 & 0.480 & 0.558 & 0.553 \\
\hline & $\mathrm{P}$ & $<0.001$ & $<0.001$ & $<0.001$ & $<0.001$ \\
\hline \multirow[t]{2}{*}{ Stair-climbing score } & $r$ & 0.629 & 0.425 & 0.520 & 0.507 \\
\hline & $\mathrm{P}$ & $<0.001$ & $<0.001$ & $<0.001$ & $<0.001$ \\
\hline \multicolumn{6}{|l|}{ Treadmill test } \\
\hline \multirow[t]{2}{*}{ PFWD - distance score } & $r$ & 0.421 & 0.380 & 0.358 & 0.632 \\
\hline & $\mathrm{P}$ & 0.032 & 0.016 & 0.003 & $<0.001$ \\
\hline \multirow[t]{2}{*}{ Speed score } & $r$ & 0.881 & 0.874 & 0.876 & 0.834 \\
\hline & $\mathrm{P}$ & $<0.001$ & $<0.001$ & $<0.001$ & $<0.001$ \\
\hline \multirow[t]{2}{*}{ Stair-climbing score } & $r$ & 0.825 & 0.800 & 0.814 & 0.805 \\
\hline & $\mathrm{P}$ & $<0.001$ & $<0.001$ & $<0.001$ & $<0.001$ \\
\hline \multirow[t]{2}{*}{ MWD - distance score } & $r$ & 0.287 & 0.321 & 0.226 & 0.538 \\
\hline & $\mathrm{P}$ & 0.165 & 0.050 & 0.075 & 0.004 \\
\hline \multirow[t]{2}{*}{ Speed score } & $r$ & 0.860 & 0.546 & 0.602 & 0.811 \\
\hline & $\mathrm{P}$ & $<0.001$ & $<0.001$ & $<0.001$ & $<0.001$ \\
\hline \multirow[t]{2}{*}{ Stair-climbing score } & $r$ & 0.761 & 0.619 & 0.642 & 0.767 \\
\hline & $\mathrm{P}$ & $<0.001$ & $<0.001$ & $<0.001$ & $<0.001$ \\
\hline
\end{tabular}

WIQ, walking impairment questionnaire; $A B I$, ankle-brachial index; PFWD, pain-free walking distance; MWD, maximum walking distance.

*Pearson correlation efficient (r) and P-value. ${ }^{\text {a) }}$ The surgery group included the bypass and intervention groups. 
also significantly improved in surgery group as compared to the medication group $(\mathrm{P}<0.001, \mathrm{P}=0.036$, and $\mathrm{P}=0.019$ for ABI, PFWD, and MWD, respectively). The WIQ score was well correlated with the objective indices in all treatment groups. Although improvements in walking ability as a result of each treatment were evident, our main focus was to verify whether the WIQ score could reflect the objective indices at each timepoint, not the effects comparison among the treatments. Importantly, this study validated the value of Korean version of the WIQ in Korean PAD patients. We noted that the WIQ could objectively and effectively indicate the patient's status throughout the study period in different clinical settings. These results suggest that the Korean WIQ can be used as an alternative test, instead of other objective examinations such as ABI or treadmill tests. Moreover, the WIQ effectively reflects the patient's daily walking ability. Thus, it offers considerable benefit in the outpatient clinic for physicians or surgeons in terms of its cost-effective and time-saving nature. Hence, the treadmill test can be performed in only selected patients.

This study has some unique characteristics as compared to previous studies on questionnaires in PAD patients. First, the Korean WIQ is reproducible and reliable in various treatment groups, including patients receiving medication, intervention, or bypass treatments. Hence, this Korean WIQ can be used in PAD patients in various clinical settings. Second, in another Korean version of a peripheral artery questionnaire suggested by Lee et al. [23], the questions were mainly focused on the patient's satisfaction with treatment, discomfort, or mood change due to the disease, whereas validity was assessed by identifying associations with other questionnaires. The method of validation, involving comparisons with other type of questionnaires, has often been used in other language versions of the WIQ $[14,17]$. However, in the present study, we directly compared the questionnaire with objective tests such as the ABI or treadmill tests. Finally, we observed that the domain scores of the Korean WIQ also reflected the ABI and the treadmill test scores. Thus, not only the total score, but also the domain scores of distance, speed, and stair climbing, of this Korean WIQ are reliable.

This study has certain limitations. First, the sample size was small due to limitations of time and funding. However, in most of the groups, we could confirm the presence of significant correlations. Second, this study did not include other PAD lesions including Buerger's disease. Third, we did not compare the WIQ with other previously developed questionnaires that often target the emotional aspects of the patients. However, we focused on whether the patient self-reported questionnaire could reflect the objective indices. Finally, this study included native Korean PAD patients only, and hence, these results may differ from those with a different ethnic background.

In conclusion, the Korean version of the WIQ appears to be valid and reproducible. These results suggest that our Korean WIQ could be effectively used to assess Korean patients with peripheral arterial disease. Therefore the WIQ can be easy and simple alternative test of the conventional tests like ABI or treadmill test for Korean IC patients and is also expected to represent the walking ability of patient's daily life more effectively.

\section{CONFLICTS OF INTEREST}

No potential conflict of interest relevant to this article was reported.

\section{ACKNOWLEDGEMENTS}

This study was supported by generous unconditional funding from Astellas Pharma Korea, Inc. (Seoul, Korea).

\section{SUPPLEMENTARY MATERIAL}

Supplementary material can be found via https://astr.or.kr/ src/sm/astr-93-103-s002.pdf

\section{REFERENCES}

1. Malgor RD, Alahdab F, Elraiyah TA, Rizvi AZ, Lane MA, Prokop LJ, et al. A systematic review of treatment of intermittent claudication in the lower extremities. J Vasc Surg 2015;61(3 Suppl): 54S-73S.

2. Schorr EN, Treat-Jacobson D. Methods of symptom evaluation and their impact on peripheral artery disease (PAD) symptom prevalence: a review. Vasc Med 2013;18:95111.

3. Rose GA, Blackburn H. Cardiovascular survey methods. Monogr Ser World Health Organ 1968;56:1-188.

4. Rose G, McCartney P, Reid DD. Self-administration of a questionnaire on chest pain and intermittent claudication. $\mathrm{Br} \mathrm{J}$ Prev Soc Med 1977;31:42-8.

5. Leng GC, Fowkes FG. The Edinburgh Claudication Questionnaire: an improved version of the WHO/Rose Questionnaire for use in epidemiological surveys. J Clin Epidemiol 1992;45:1101-9.

6. Criqui MH, Denenberg JO, Bird CE, 
Fronek A, Klauber MR, Langer RD. The correlation between symptoms and noninvasive test results in patients referred for peripheral arterial disease testing. Vasc Med 1996;1:65-71.

7. EuroQol Group. EuroQol: a new facility for the measurement of health-related quality of life. Health Policy 1990;16:199208.

8. Brooks R. EuroQol: the current state of play. Health Policy 1996;37:53-72.

9. Frendl DM, Ware JE Jr. Patient-reported functional health and well-being outcomes with drug therapy: a systematic review of randomized trials using the SF36 health survey. Med Care 2014:52:43945.

10. Hiatt WR, Rogers RK, Brass EP. The treadmill is a better functional test than the 6-minute walk test in therapeutic trials of patients with peripheral artery disease. Circulation 2014:130:69-78.

11. Regensteiner JG, Steiner JF, Panzer RJ, Hiatt WR. Evaluation of walking impairment by questionnaire in patients with peripheral arterial disease. J Vasc Med Biol 1990;2:142-52.

12. Hiatt WR, Hirsch AT, Regensteiner JG, Brass EP. Clinical trials for claudication. Assessment of exercise performance, functional status, and clinical end points.
Vascular Clinical Trialists. Circulation 1995:92:614-21.

13. Norgren L, Hiatt WR, Dormandy JA, Nehler MR, Harris KA, Fowkes FG, et al. Inter-Society Consensus for the Management of Peripheral Arterial Disease (TASC II). Eur J Vasc Endovasc Surg 2007;33 Suppl 1:S1-75.

14. Verspaget M, Nicolaï SP, Kruidenier LM, Welten RJ, Prins MH, Teijink JA. Validation of the Dutch version of the Walking Impairment Questionnaire. Eur J Vasc Endovasc Surg 2009;37:56-61.

15. Wohlgemuth WA, Niechzial M, Nagel E, Bohndorf $\mathrm{K}$. Assessment of the quality of life of patients with peripheral vascular diseases. Rofo 2003;175:169-75.

16. Schiano V, Brevetti G, Sirico G, Silvestro A, Giugliano G, Chiariello M. Functional status measured by walking impairment questionnaire and cardiovascular risk prediction in peripheral arterial disease: results of the Peripheral Arteriopathy and Cardiovascular Events (PACE) study. Vasc Med 2006;11:147-54.

17. Lozano FS, March JR, González-Porras JR, Carrasco E, Lobos JM, Areitio-Aurtena A. Validation of the Walking Impairment Questionnaire for Spanish patients. Vasa 2013:42:350-6.

18. Sigvant B, Lundin F, Nilsson B, Bergqvist D,
Wahlberg E. Differences in presentation of symptoms between women and men with intermittent claudication. BMC Cardiovasc Disord 2011;11:39.

19. Matsuo H, Shigematsu H. Patient-based outcomes using the Walking Impairment Questionnaire for patients with peripheral arterial occlusive disease treated with Lipo-PGE1. Circ J 2010;74:365-70.

20. Yan BP, Lau JY, Yu CM, Au K, Chan KW, Yu DS, et al. Chinese translation and validation of the Walking Impairment Questionnaire in patients with peripheral artery disease. Vasc Med 2011;16:167-72.

21. Beaton DE, Bombardier C, Guillemin F, Ferraz MB. Guidelines for the process of cross-cultural adaptation of selfreport measures. Spine (Phila Pa 1976) 2000;25:3186-91.

22. Jie Wang, Yan Cui, Bian RW, Mo YZ, Haidi $\mathrm{Wu}$, Ling Chen. Validation of the Chinese version of the Walking Impairment Questionnaire in patients with both peripheral arterial disease and type 2 diabetes mellitus. Diab Vasc Dis Res 2011;8:29-34.

23. Lee JH, Cho KI, Spertus J, Kim SM. Crosscultural adaptation and validation of the Peripheral Artery Questionnaire: Korean version for patients with peripheral vascular diseases. Vasc Med 2012;17:215-22. 